\title{
THE ACCESS CONTROL SYSTEM BASED ON LINEAR DISCRIMINANT ANALYSIS
}

\begin{abstract}
Widodo Budiharto
Department of Informatics Engineering, School of Computer Science, Bina Nusantara University, Jakarta, Indonesia

Received 2013-09-02; Revised 2013-10-08; Accepted 2013-11-29

ABSTRACT

Face recognition represents an important computer vision domain that has been researched in the last decades. The objective of this research is to develop an Access Control System based on Face Recognition using Linear Discriminant Analysis (LDA) method. The analysis is done by making an application of face recognition using LDA for extracting features of the face and the output for controlling relay as a simulation of a door using AVR Microcontroller. The result achieved by the application is a face recognition system using LDA can obtain excellent result that is $95 \%$ success rate in face recognition with the image of a face that was tested by 40 people, instead of the previously popular feature extraction methods such as PCA. Based on the experimental results, attendance system based on face recognition using LDA obtain better result compared with PCA based face recognition.
\end{abstract}

Keywords: Access Control System, LDA, Face Recognition

\section{INTRODUCTION}

Face recognition represents a very important biometric domain, the human face being a psychological biometric identifier that is widely used in person authentication. A facial recognition system represents a computer-driven application for automatically authenticating a person from a digital image, using the characteristics of its face. Face recognition technologies have a variety of application areas, such as: Attendance and access control systems for various services, surveillance systems and law enforcement (Barbu, 2011).

Access control system is very important and used in workplaces and educations. The absence system has made an evolution from manual and simple absence system, such as call name, raise hands at school to automatic and complex absence system, such as print the arrival time or tap a specific card that have been used recently in modern university or company. The evolution of absence system is happened deliberately to ease the stakeholder control and manage a lot of people that is hard to be done if we still use manual and simple way. But, the evolution in absence system is not as effective as it was planned before. There are some misuses and cheats in running absence system in the real world. Entrustment of absence card and signature are few examples that are often done by students or employees to have their names be recorded, but they don't actually come. The implementation of absence system is still need improvement to minimalize cheats and tricks so absence system can be used as it was planned before, to keep record a large number of students or employees.

Face recognition is an improvement an ordinary face detection which makes only a computer to detect any faces by using webcam as an example. Face recognition system requires face detection and face recognition as the basic steps for complete. By applying a complete face recognition system, violations can be reduced and even be eliminated. By applying face recognition system integrated with absence system, there will be no chance for students to entrust their ID card to friends or another person, because there will be a webcam to capture their face and match it with an image in the database that has been saved before.

There are some methods for face recognition, such as PCA, LDA, Nearest features classification and combined with neural network such as PCA-ANN (Mario, 2009). But, what we choose is Linear Discriminant Analysis 
(LDA), which is effective. Later on, we will also prove that using LDA is better than using PCA. We will try to compare the accuracy of LDA with the accuracy of PCA that has already been tested before.

\section{MATERIALS AND METHODS}

\subsection{Computer Vision}

Computer Vision is a science that study how computer can recognize detected object (Szeliski, 2010). Computer Vision is the combination of image processing and pattern recognition. Computer Vision with Artificial Intelligence will be able to produce visual intelligence system. Face recognition is a task that routinely and easily worked by human in daily activities. Face recognition research and development have been improved automatically with powerful desktop availability and low cost and embedded-system which have created a big interest in digital and video image processing. The motivation and development of the research are included in biometric authentication subspace, surveillance, human-computer interaction and multimedia management. Although the digital image processing filed is built on a foundation of mathematical and probabilistic formulations, human instituition and analysis play a central role in visual judgements (Gonzales and Woods, 2009).

\subsection{Linear Discriminant Analysis (LDA)}

It was firstly developed by an English statistician, Sir Ronald Aylmer Fisher (R.A Fisher) in 1936. It was also called Fisher Linear Discriminant in another word and a classic method for feature extraction used in face recognition. LDA is used widely to find out the linear combinations of features while preserve class separability. Linear Discriminant Analysis (LDA) often produces model that the accuracy approaches (sometimes exceeds) more complex modern methods. Discriminant analysis can be used for nothing but for classification. In linear discriminant analysis of statistics, within-class, between-class and mixture scatter matrices are used to formulate criteria of class separability (Bostjan et al., 2012; Belhumeur et al., 1997).

The algorithm of fisherfaces (LDA) is defined as.

For example, $\mathrm{X}_{\mathrm{i}}$ is a random vector taken from class C Equation (1):

$$
\mathrm{X}=\left\{\mathrm{X}_{1}, \mathrm{X}_{2}, \ldots \mathrm{X}_{\mathrm{C}}\right\}
$$

Scatter matrix between $\left(S_{B}\right)$ and within $\left(S_{W}\right)$ are calculated as Equation (2 and 3):

$$
\begin{aligned}
& S_{B}=\sum_{i=1}^{C} N_{i}\left(\mu_{i}-\mu\right)\left(\mu_{i}-\mu\right)^{T} \\
& S_{W}=\sum_{i=1}^{\mathrm{C}} \sum_{x j X i}\left(\mu_{j}-\mu_{i}\right)\left(\mu_{j}-\mu_{i}\right)^{T}
\end{aligned}
$$

Where:

$\mu_{\mathrm{i}}=$ Mean from all class $X_{\mathrm{i}}$

$\mu=$ Mean from all class $C$

$\mathrm{X}_{\mathrm{j}}=$ Sample vector $\mathrm{j}$ from different class $\mathrm{X}_{\mathrm{i}}$

$\mathrm{N}_{\mathrm{i}}=$ Sample at class $\mathrm{X}_{\mathrm{i}}$

where, $\mu$ is the global Equation (4):

$\mu_{\mathrm{i}}=\frac{1}{\mathrm{~N}} \sum_{\mathrm{i}=1}^{\mathrm{N}} \mathrm{x}_{\mathrm{i}}$

Meanwhile $\mu$ is the mean from classi $\in\{1, \ldots, C\}$ Equation (5):

$\mu_{\mathrm{i}}=\frac{1}{\left|\mathrm{X}_{\mathrm{i}}\right|} \sum_{\mathrm{Xj \in XiXj}}$

Later, we seek projection $\mathrm{W}$ that maximize class separability criterion Equation (6 and 7):

$$
\begin{aligned}
& \mathrm{W}_{\mathrm{opt}}=\arg \max _{\mathrm{w}} \frac{\left|\mathrm{W}^{\mathrm{T}} \mathrm{S}_{\mathrm{B}} \mathrm{W}\right|}{\left|\mathrm{W}^{\mathrm{T}} \mathrm{S}_{\mathrm{w}} \mathrm{W}\right|} \\
& \mathrm{W}_{\mathrm{opt}}=\left[\mathrm{w}_{1} \mathrm{w}_{2} . . \mathrm{w}_{\mathrm{m}}\right]
\end{aligned}
$$

where, $\mathrm{W}_{\mathrm{opt}}=$ Class separability criterion.

If $\mathrm{S}_{\mathrm{W}}$ is nonsingular, optimum projection is chosen from matrix with orthonormal column which maximalizes ratio from determinant between-class scatter matrix and within-class scatter matrix. Where $\left\{\mathrm{W}_{\mathrm{i}} \mid \mathrm{i}=1,2, \ldots \mathrm{m}\right\}$ is a generalization of eigenvector from $S_{B}$ and $S_{W}$ that correspond with biggest $\mathrm{m}$ of General Eigenvalue. optimization problem solution was given by finishing general eigenvalue problem Equation (8 and 9):

$$
\begin{aligned}
& \mathrm{S}_{\mathrm{B}} \mathrm{W}_{\mathrm{i}}=\lambda \mathrm{S}_{\mathrm{W}} \mathrm{W}_{\mathrm{i}} \\
& \mathrm{S}_{\mathrm{W}}^{-1} \mathrm{~S}_{\mathrm{B}} \mathrm{W}_{\mathrm{i}}=\lambda_{\mathrm{i}} \mathrm{W}_{\mathrm{i}}
\end{aligned}
$$

where, $i=1,2, \ldots, m \lambda$ eigenvalue. Example of LDA is shown in Fig. 1. 

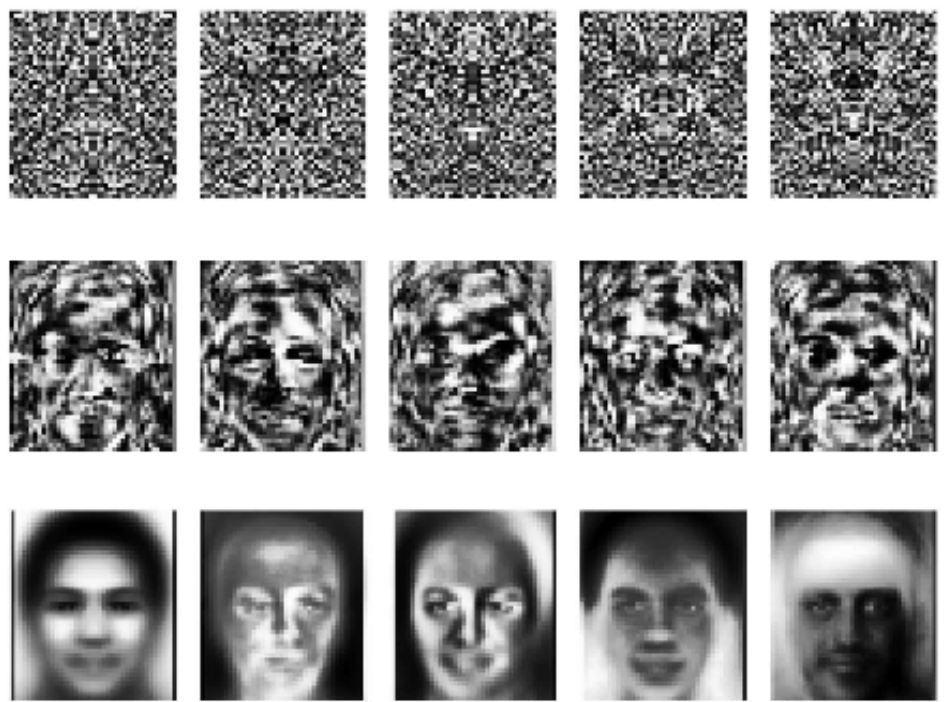

Fig. 1. Different projection bases constructed from a set of 444 individuals, where the set is augumented via adding noise and mirroring. The first row shows the first five pure LDA basis images W; the second row shows the first five subspace LDA basis images $\mathrm{W} \phi$; the average face and first four eigenfaces $\phi$ are shown on the third row

The remaining problem is $\mathrm{S}_{\mathrm{W}}$ has many $(\mathrm{N}-\mathrm{C})$, with sample $\mathrm{N}$ and class $\mathrm{C}$. In pattern recognition, sample $\mathrm{N}$ always fewer than input data dimension (number of pixels), which cause matrix $S_{\mathrm{W}}$ becomes singular Fisherfacesor LDA can be performed to reduced data because $S_{\mathrm{W}}$ is not singular anymore. The problem can now be written as Equation (10 and 11):

$\mathrm{W}_{\mathrm{pca}}=\arg \max _{\mathrm{w}}\left|\mathrm{W}^{\mathrm{T}} \mathrm{S}_{\mathrm{T}} \mathrm{W}\right|$

$\mathrm{W}_{\text {fld }}=\arg \max _{\mathrm{w}} \frac{\left|\mathrm{W}^{\mathrm{T}} \mathrm{W}_{\mathrm{pca}}^{\mathrm{T}} \mathrm{S}_{\mathrm{B}} \mathrm{W}_{\mathrm{pca}} \mathrm{W}\right|}{\left|\mathrm{W}^{\mathrm{T}} \mathrm{W}_{\mathrm{pca}}^{\mathrm{T}} \mathrm{S}_{\mathrm{W}} \mathrm{W}_{\mathrm{pca}} \mathrm{W}\right|}$

Transformation matrix $\mathrm{W}$ which projects sample to dimension (C-1) defined as Equation (12):

$\mathrm{W}=\mathrm{W}_{\mathrm{fld}}^{\mathrm{T}} \mathrm{W}_{\mathrm{pca}}^{\mathrm{T}}$

Besides applied in training data set, transformation is also applied in testing data set. Figure 1 will represents results of face images that shows discriminatory power of whole image is significantly larger than any discriminatory power of any parts of face.

\subsection{Euclidean Distance}

Euclidean distance the most common use of distance. It examines the root of square differences between coordinates of a pair of object (Belhumeur et al., 1997). The pattern of Euclidean distanceis Equation (14):

$$
\mathrm{D}_{\mathrm{ij}}=\sqrt{\sum_{\mathrm{k}=1}^{\mathrm{n}}\left(\mu_{\mathrm{ik}}-\mu_{\mathrm{jk}}\right)^{2}}
$$

\subsection{Proposed Method}

We had develop an Attendance system using webcam and AVR Microcontroller minimum system with LCD Display before. AVR is a low cost microcontroller that suitable for general purpose application. Software used by the PC are open CV and Visual Studio 2010 Express edition. Webcam as a main sensor, will capture the user's face in front of a camera to compare with the data contained in the face database. If the face in front of the camera recognized, then the relay is active and the door will be opened. Figure 2 below show the framework of the system.

\section{RESULTS}

We use webcam and Laptop using OpenCV to conduct the experiment. Figure 3 below shows that the face has no name and-1 value because he doesn't belong to any class since he has not been added to database. Figure 4 below shows how to input your name when your face want to be added to database. Figure 5 below shows that the system able to identify face based on the training set. 


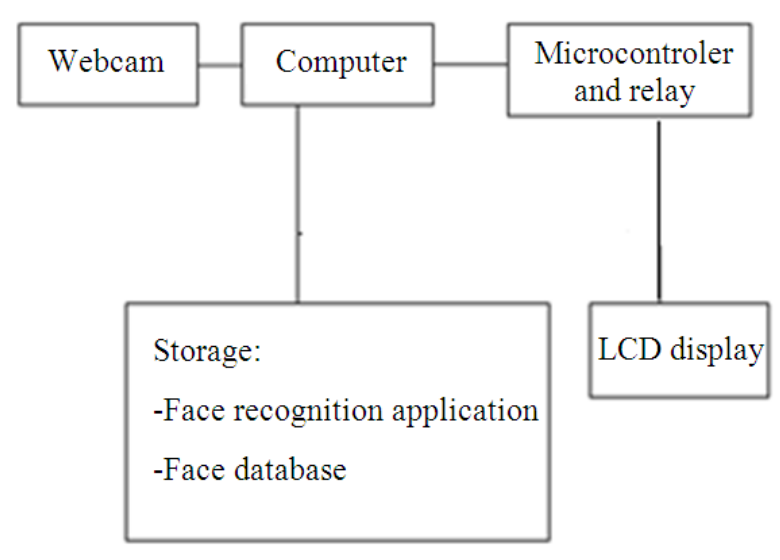

Fig. 2. A framework of the access control system

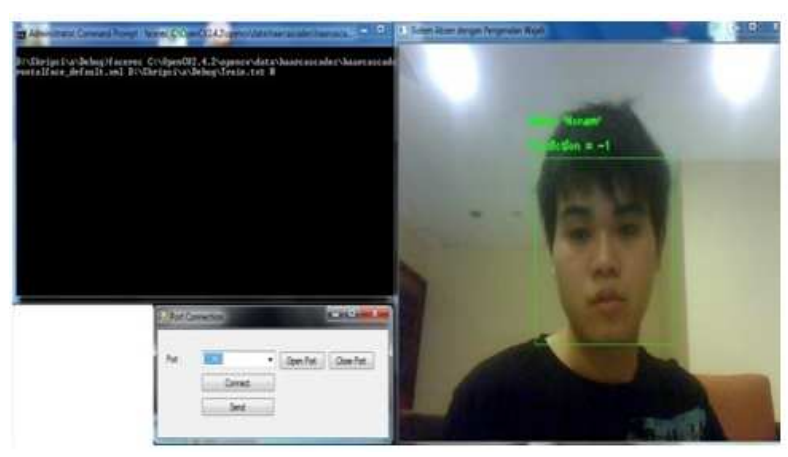

Fig. 3. Result of a program for the first time

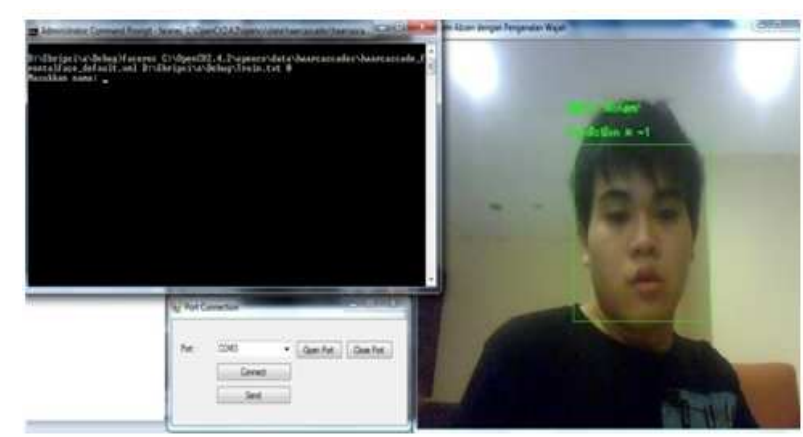

Fig. 4. User must add his name as a new data

Table 1 shows that the more image tested, the more time it takes. It also shows that the more image tested, the less accuracy it gets by using AT and T database.

Figure 6 show our minimum system of AVR microcontroller to activate/deactive relay.

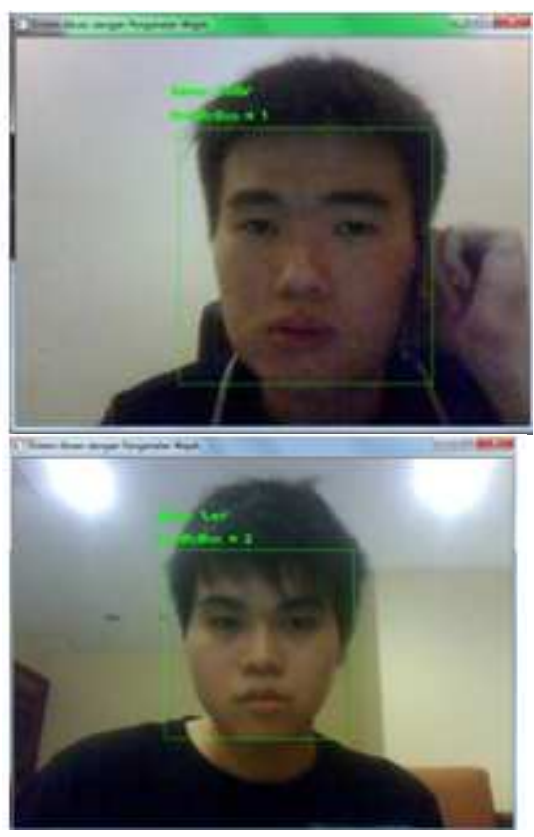

Fig. 5. All faces in training set have been recognized successfully with right prediction labels and names If the face recognized, the program will send data through USB port to AVR Microcontroller to activate the relay, as a simulation for a door

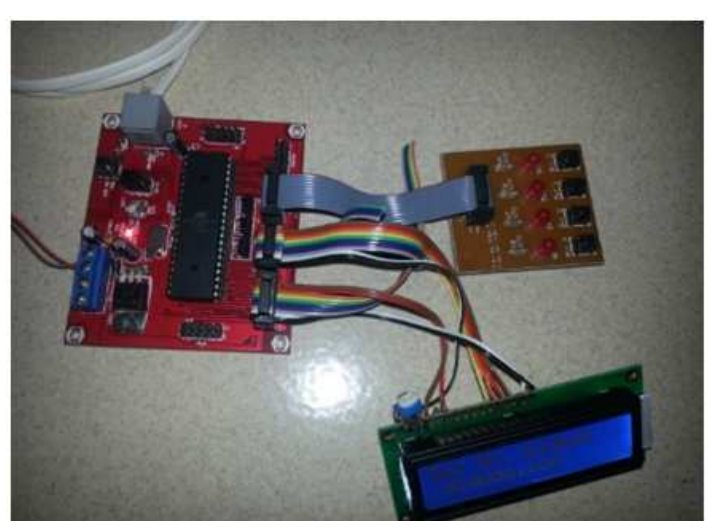

Fig. 6. AVR Microcontroller connected to USB port and relay used as a simulation for a door. If face recognized, then the relay of the door is opened

\section{DISCUSSION}

Experiments have been performed to test the proposed method. Microsoft Visual $\mathrm{C}++2010$ Express is the software tool that were used for this experiment. Table 1 give the result of evaluation testing set which reach the accuracy between $95-100 \%$. 
Table 1. Evaluation testing set

\begin{tabular}{lll}
\hline Testing set & Time $(\mathrm{sec})$ & Accuracy\% \\
\hline 10 & 6 & 100 \\
20 & 40,7 & 100 \\
40 & 81,2 & 95 \\
\hline
\end{tabular}

Table 2. Evaluation training set

\begin{tabular}{ll}
\hline Training set & Time (sec) \\
\hline 50 & 6 \\
100 & 40,7 \\
200 & 274,5 \\
\hline
\end{tabular}

Table 2 shows that the more images in training set, the more time it takes to completing the face recognition application by using AT and T database. But overall, the time needed for recognize the face is enough fast.

\section{CONCLUSION}

Using face recognition system as an access control system is very useful and can help universities track their students. There will be no more violation that often happens when manual systems are used. Face recognition can ensure the students not to entrust the ID card or misuse the ID card if modern system of absence is applied. Face recognition system will detect students' face personally and later on, match it with the image that has already been recorded before in database to give permissions correctly to students to get in to the classroom. Face recognition system will definitely help universities to run absence systems easily and automatically. By applying LDA as method for face recognition is more efficient than any other method because the accuracy of correct matches is much better.

\section{REFERENCES}

Barbu, T., 2011. Face analysis, modeling and recognition systems. InTech-Open Access Company.

Belhumeur, P., J.P. Hespanha and D.J. Kriegman, 1997. Eigenfaces Vs. Fisherfaces: Recognition using class specific linear projection. IEEE Trans. Patt. Anal. Mach. Intell., 19: 711-720. DOI: 10.1109/34.598228

Bostjan, V., J.Z. Gros, N. Pavesic and V. Struc, 2012. Face recognition using simplified probabilistic linear discriminant analysis. Int. J. Adv. Robot. Syst., 9: 180-180. DOI: $10.5772 / 52258$

Gonzales, R.C. and R.E. Woods, 2009. Digital Image Processing. 1st Edn., Pearson Education India, UP, India, ISBN-10: 8131726959, pp: 954.

Mario, I., 2009. State of the Art in Face Recognition. ITech Education and Publishing.

Szeliski, R., 2010. Computer Vision: Algorithms and Applications. 1st Edn., Springer, London, ISBN-10: 1848829353, pp: 832. 